\begin{tabular}{|c|l|}
\hline Title & Exciton-exciton interaction and heterobiexcitons in GaN \\
\hline Author(s) & A dachi, S.; Muto, S.; Hazu, K.; Suzuki, K.; Sota, T.; Chichibu, S. F.; Mukai, T. \\
\hline Citation & $\begin{array}{l}\text { PHY SICAL REVIEW B, 67, 205212 } \\
\text { https://doi.org/L0.1103/PhysRevB.67.205212 }\end{array}$ \\
\hline Issue Date & 2003 \\
\hline Doc URL & http://hdl.handle.net/2115/5784 \\
\hline Rights & Copyright $\odot 2003$ A merican Institute of Physics \\
\hline Type & article \\
\hline File Information & PRB67.pdf \\
\hline
\end{tabular}

Instructions for use 


\title{
Exciton-exciton interaction and heterobiexcitons in GaN
}

\author{
S. Adachi ${ }^{*}$ and S. Muto ${ }^{\dagger}$ \\ Department of Applied Physics, Hokkaido University, Kitaku, Sapporo 060-8628, Japan \\ K. Hazu, T. Sota, ${ }^{\ddagger}$ and K. Suzuki \\ Department of Electrical, Electronics, and Computer Engineering, Waseda University Shinjuku, Tokyo 169-8555, Japan \\ S. F. Chichibu ${ }^{\S}$ \\ Institute of Applied Physics; Graduate School of Pure and Applied Sciences, University of Tsukuba, 1-1-1 Tennodai, Tsukuba, \\ Ibaraki 305-8573, Japan \\ T. Mukai \\ Nitride Semiconductor Research Laboratory, Nichia Corporation, Oka, Kaminaka, Anan, Tokushima 744-8601, Japan
}

(Received 31 October 2002; published 28 May 2003)

\begin{abstract}
The formation of not only $A$ biexcitons $\left(X X_{A A}\right)$ but also heterobiexcitons that consist of $A$ and $B$ excitons $\left(X X_{A B}\right)$ in a free-standing bulk $\mathrm{GaN}$ is identified by polarization-sensitive spectrally resolved FWM measurements. The FWM spectra and delay-time dependence show that the interaction between $A$ and $B$ exciton gives rise to the energy shifts of the spectra and the phase shifts of the quantum beating, which is considered as the effect of the unbound state of $X X_{A B}$ (i.e., $X X_{A B}^{*}$ ) and $X X_{A B}^{*}$ is found to play an important role in the FWM signals for all polarizations. The unbound $A$ biexciton $\left(X X_{A A}^{*}\right)$ is also observed clearly in spectral and temporal domains.
\end{abstract}

DOI: 10.1103/PhysRevB.67.205212

PACS number(s): 42.50.Md, 71.55.Eq, 71.35.Cc, 78.47.+p

\section{INTRODUCTION}

Coherent nonlinear optical processes involving bound biexcitons $(X X)$ have been investigated extensively in bulk and quantum wells. Biexciton effects associated with $X X$ have been identified even in III-V semiconductors and more easily in II-VI and I-VII semiconductors. However, processes involving two excitons require obviously to take account of four-particle correlations, ${ }^{1-8}$ which mean not only $X X$ but also unbound biexciton $\left(X X^{*}\right)$. Actually, $X X^{*}$ can affect the observed signals strongly even under no clear signature of $X X$, for example, in cocircularly polarized excitation where $X X$ cannot be created according to the polarization selection rules. Recently, it was found that excitonexciton correlations can have important, and even dominant, effects at low density by Kner et al. ${ }^{8}$ They used the spatial confinement by magnetic field to enhance the strength of the exciton-exciton correlations. In the sense, exciton-biexciton system is an appropriate system to the investigation of manyparticle correlations that are rarely accessed directly, where the scattering processes of unbound biexciton $X X^{*}$ as well as $X X$ and their correlation with the exciton scattering processes is of considerable current interest.

Gallium nitride $(\mathrm{GaN})$ has large exciton binding energies of more than $20 \mathrm{meV}$ because of the relatively small dielectric constant and large effective masses, and therefore, the large binding energies of biexcitons are expected. The large binding energies and strong optical nonlinearities make it suitable material for the study of biexcitons and Coulomb correlations of their excitons by using the FWM technique. Recently, the degenerate FWM has been applied to study the properties of excitons ${ }^{9-12}$ and biexcitons ${ }^{13,14}$ in bulk GaN. The biexcitonic contribution to the FWM signals may appear strongly in most usual excitation conditions by ultrashort pulses, and therefore is very important. But it is little known currently about the Coulomb correlations including the information of the bound biexcitons in GaN.

In this paper, we investigate experimentally the bound and unbound biexciton contributions to the spectrally resolved FWM signals in a free-standing bulk GaN. The spectra of heterobiexciton $X X_{A B}$ that consists of $A$ - and $B$-hole excitons as well as $A$-hole bound $\left(X X_{A A}\right)$ and unbound $\left(X X_{A A}^{*}\right)$ biexcitons were clearly observed. Observation of $X X_{A B}$ in $\mathrm{GaN}$ is for the first time to our best knowledge. The unbound $X X_{A B}^{*}$ is essential for the $X_{A}-X_{B}$ interaction and contributes more significantly to the FWM signal generation rather than the bound $X X_{A B}$ biexcitons.

\section{SAMPLE AND EXPERIMENT}

The investigated sample is a free-standing $c$-face wurtzite GaN of $70-\mu \mathrm{m}$ thickness by the two-flow metal-organic chemical vapor deposition method ${ }^{15}$ using the lateral epitaxial overgrowth technique. ${ }^{16,17} \mathrm{GaN}$ crystallizes in the wurtzite structure, whose valence bands consist of $A, B$, and $C$ bands that are split each other even at $\Gamma$ point due to the crystal field and spin-orbit interaction. ${ }^{18}$ Therefore, the corresponding exciton structure consists of $A, B$, and $C$ excitons (denoted hereafter as $X_{A}, X_{B}$, and $X_{C}$, respectively). The transition energy difference between $X_{A}$ and $X_{B}$ is known to be $5-6 \mathrm{meV}$, and the $X_{C}$ resonance is apart by more than 10 $\mathrm{meV}$ from the $X_{B}$ resonance.

The spectrally resolved, time-integrated two-pulse FWM experiments in reflection geometry are performed with the excitation pulses of the same intensities in the directions $\boldsymbol{k}_{1}$ and $\boldsymbol{k}_{2}$, respectively. The pulses from a frequency-doubled, 
mode-locked Ti-doped sapphire laser with the spectral width of $17.5 \mathrm{meV}$ (FWHM) is used as a light source. The emitted FWM signal in the $2 \boldsymbol{k}_{2}-\boldsymbol{k}_{1}$ direction is spatially selected by an iris, spectrally resolved by a spectrometer with the resolution of $0.9 \mathrm{meV}$ (FWHM), and detected phase sensitively by a photomultiplier. The delay time $\tau_{12}$ between the two incident pulses is defined to be positive if the $\boldsymbol{k}_{1}$ pulse precedes the $\boldsymbol{k}_{2}$ pulse. The total excitation intensity is $140 \mathrm{~nJ} / \mathrm{cm}^{2}$, corresponding to the excited exciton densities of $\sim 10^{16} \mathrm{~cm}^{-3}$, which is two orders of magnitude smaller than the screening density $\left(\sim 2 \times 10^{18} \mathrm{~cm}^{-3}\right)$. The sample is placed in a closed-cycle helium cryostat and all presented FWM data are taken at $10 \mathrm{~K}$. The detail of the experimental setup is seen in Ref. 12 .

\section{RESULTS AND DISCUSSIONS}

Polarization selection rules in FWM can be used to discriminate transitions from the vacuum state $(G)$ to one of the optically active exciton states $(X)$ or transitions from $X$ to bound biexciton states $(X X)$ (see Fig. 2). The FWM signal originates from the nonlinearities of phase-space filling (PSF), bare Coulomb interaction (BCI), and exciton-exciton correlation (XXC), which is significantly dependent of light polarizations and temporal ordering of the excitation pulses via the parametric relation between coherently created polarizations and occupations of the involved transitions and states. Here BCI means Coulomb nonlinearities within the Hartree-Fock treatment and the interaction to induce bandedge renormalization and local-field effect. ${ }^{19}$

Figure 1(a) shows the FWM spectra at $\tau_{12}=0.8$ ps and for different polarization configurations. The labels $X_{B}, X_{A}$, and $X X_{A A}$ represent the $B$-exciton, $A$-exciton, and the bound $A$-biexciton states, respectively. The arrows on the top axis point to the energies of the transverse $X_{A}(3.4791 \mathrm{eV})$ and $X_{B}$ $(3.4844 \mathrm{eV})$ resonances that were obtained from the analysis of the reflection spectra. ${ }^{20}$ Thus, $X_{A}-X X_{A A}$ corresponds to the transition energy from $X_{A}$ to $X X_{A A}$. From the position of $X_{A}-X X_{A A}$, the $X X_{A A}$-binding energy is found to be $5.3 \mathrm{meV}$. We have tuned the center laser wavelength energetically below the $X_{A}$ resonance as shown in Fig. 1(a), in order to minimize the excitation of $X_{C}$ and exciton continua. $X_{C}$ resonance locates by $18.3 \mathrm{meV}$ above $X_{B}$ resonance in this sample. The transition matrix element $\mu_{A}$ of $X_{A}$ is larger by a small amount than $\mu_{B}$ of $X_{B}$ (the calculated ratio $\left.\mu_{A}^{2}: \mu_{B}^{2} \sim 1: 0.88\right)$ and the FWM signals are proportional to $N_{i}^{2} \mu_{i}^{8}(i=A, B)$, where $N_{i}$ is the density of the corresponding exciton state. Consequently, the FWM signal at $X_{A}$ are stronger than that at $X_{B}$.

The relative contributions between $X_{A}$ and $X X_{A A}$ to the FWM signal are dependent strongly of the excitation polarization. For cocircular polarizations $\left(\sigma_{+}, \sigma_{+}\right)$, the transition from $X_{A}\left(X_{B}\right)$ to $X X_{A A}\left(X X_{B B}\right)$ is not allowed since $X X_{A A}\left(X X_{B B}\right)$ consists of two $X_{A} \mathrm{~s}\left(X_{B} \mathrm{~s}\right)$ with opposite exciton spin and has the angular momentum $J_{z}=0$. Thus, the signal $X_{A}-X X_{A A}$ does not appear in $\left(\sigma_{+}, \sigma_{+}\right)$spectra. The $X_{A}$ line broadens with relatively small inhomogeneity compared with the temporal FWM trace (Fig. 3) that decays with the time constant of $0.66 \mathrm{ps}$ for $\tau_{12}>0$. The decay constant
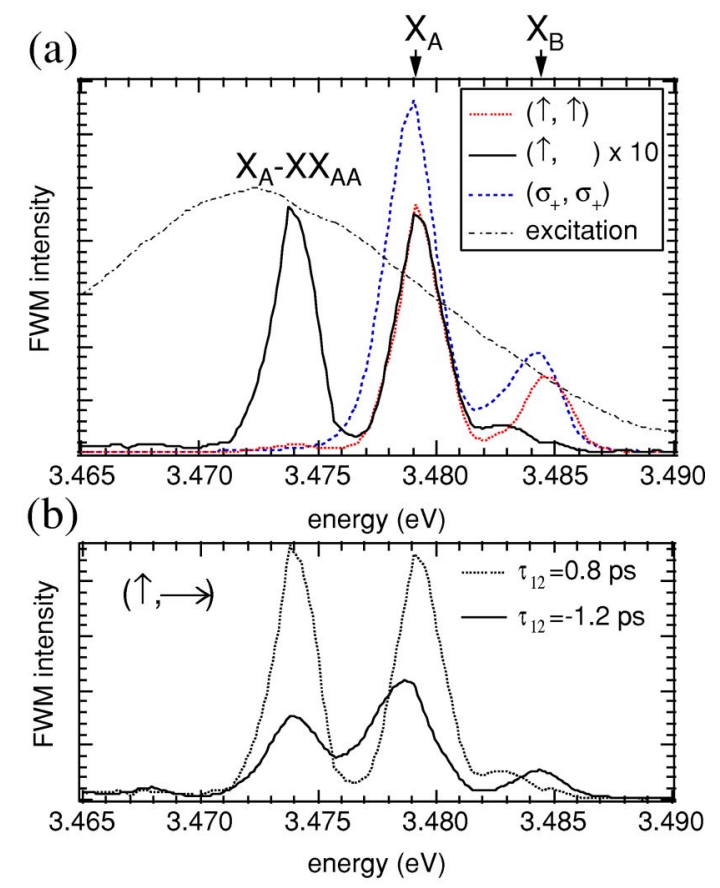

(c)

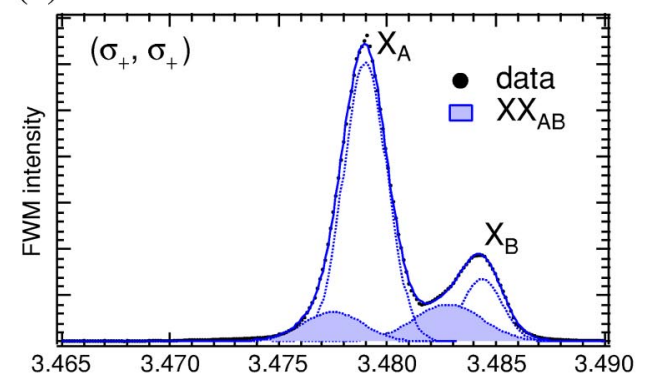

(d)

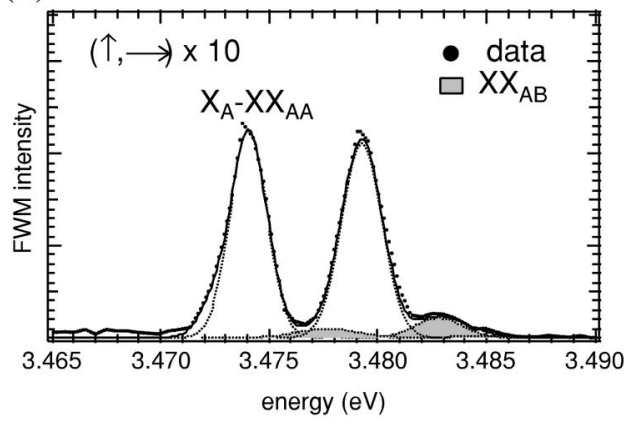

FIG. 1. (a) FWM spectra at $\tau_{12}=0.8$ ps. The three excitation polarizations are used as indicated. The spectrum in $(\uparrow, \rightarrow)$ polarizations is multiplied by 10 . The arrows at the top axis indicate the energies of $A$ - and $B$-exciton resonances obtained by the reflection spectra. The excitation spectrum is also indicated. (b) FWM spectra in $(\uparrow, \rightarrow)$ at positive $\left[\tau_{12}=0.8 \mathrm{ps}\right.$, same as (a) $]$ and negative $\left(\tau_{12}\right.$ $=-1.2 \mathrm{ps}$ ) delay times. (c) and (d) The fitting results of FWM spectra at $\tau_{12}=0.8 \mathrm{ps}$. The solid circles and dotted lines are the experimental data and the decomposed peaks. The solid line is the total fitted spectrum. The shaded components indicate the heterobiexcitons $X X_{A B}$ that consist of $X_{A}$ and $X_{B}$. Note that these components are seen at the lower-energy side of $X_{A}$ and $X_{B}$ in all polarization configurations shown in Fig. 1(a). 


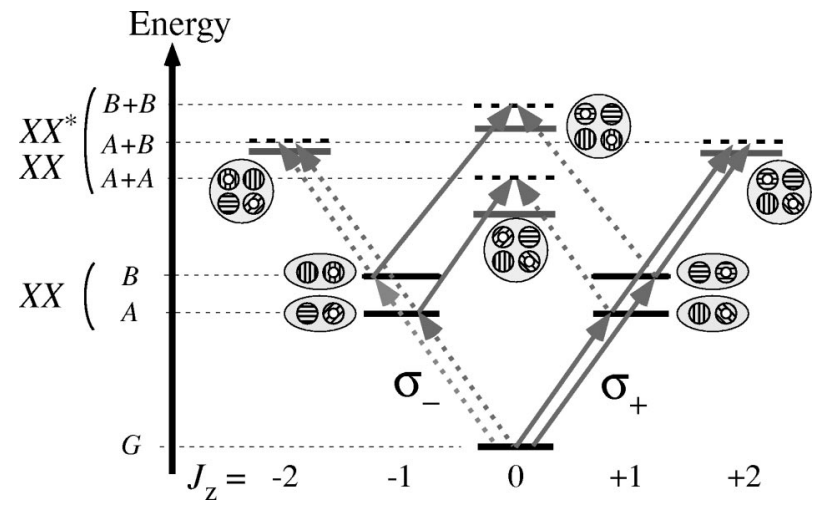

FIG. 2. Level diagram of exciton-biexciton system for a helicity basis. The thick solid lines represent exciton $X$ and bound biexciton $X X$ states to which the transitions are allowed. The thick dashed lines above the bound biexciton states indicate the corresponding biexciton continuum edge $(2 X)$. $X$ 's and $X X$ 's are illustrated with their constituent electrons and holes. Electron is depicted as a circle with vertical $\left(m_{J}^{c}=-1 / 2\right)$ or horizontal lines $\left(m_{J}^{c}=1 / 2\right)$. Hole is as a double circle with vertical $\left(m_{J}^{v}=1 / 2\right)$, horizontal $\left(m_{J}^{v}=-1 / 2\right)$, right-oblique $\left(m_{J}^{c}=3 / 2\right)$, and left-oblique $\left(m_{J}^{c}=-3 / 2\right)$ lines.

corresponds to the homogeneous broadening of $500 \mu \mathrm{eV}$ and therefore the ratio $\Gamma_{\text {inhomo }} / \Gamma_{\text {homo }}$ is found to be $\sim 4$. Taking account into the exciton lifetime and acoustic phonon scattering of this sample, ${ }^{12}$ the broadening consists of $330 \mu \mathrm{eV}$ by radiative broadening, $90 \mu \mathrm{eV}$ by acoustic phonon scattering, and $\sim 80 \mu \mathrm{eV}$ by density-dependent broadening under these experimental conditions.

For cross-linear polarization $(\uparrow, \rightarrow)$, the signals at the energies of $X_{A}$ and $X_{B}$ are strongly suppressed by the quenching of the signal generation due to excitation-induced dephasing (EID), ${ }^{21,22}$ which is a part of XXC, and the signal intensity at the energy of $X_{A}$ becomes comparable to the $X_{A}-X X_{A A}$ signal. Note that, in Fig. $1(\mathrm{a})$, the $(\uparrow, \rightarrow)$ signal is

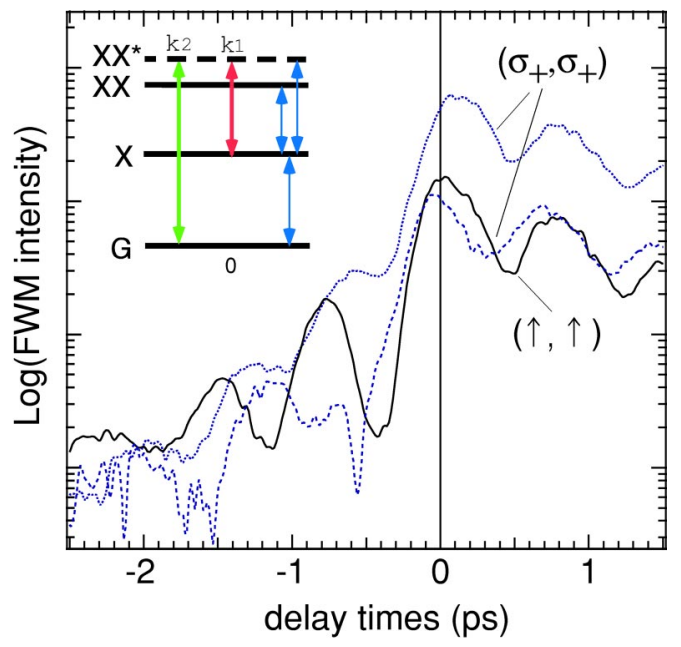

FIG. 3. Spectrally resolved FWM traces at $X_{A}$ energy as a function of $\tau_{12}$ for $\left(\sigma_{+}, \sigma_{+}\right)$at $G-X_{A}$ (dotted line), at $G-X_{B}$ (dashed line), and ( $\uparrow, \uparrow)$ at $G-X_{A}$ (solid line). Inset, a simple level diagram for the signal generation process. Note that both $X_{A}$ and $X_{B}$ are excited in the experiment. multiplied by 10 . From the polarization selection rules, the FWM signal in the direction $2 \boldsymbol{k}_{2}-\boldsymbol{k}_{1}$ for $\tau_{12}>0$ should arise from the $X_{A}-X X_{A A}$ and $X_{A}-X X_{A A}^{*}$ transitions for $(\uparrow, \longrightarrow)$. Thus, the observed FWM signal in the vicinity of the energy of $X_{A}$ is associated with the unbound biexciton $X X_{A A}^{*}$. From the fact that the transition energies $G-X_{A}$ and $X_{A}-X X_{A A}^{*}$ are the same within our spectral resolution and $X_{A}-X X_{A A}$ and $X_{A}-X X_{A A}^{*}$ transitions have the comparable strength and width that is the same as $G-X_{A}$ for $\left(\sigma_{+}, \sigma_{+}\right)$, the following are found; the unbound state $X X_{A A}^{*}$ locates at the edge of two $A$-exciton continuum, the $X_{A}-X X_{A A}^{*}$ transition has the similar magnitude of the matrix element as $X_{A}-X X_{A A}$, and the broadenings of $X_{A}, X X_{A A}$, and $X X_{A A}^{*}$ have a nearly perfect correlation. This is quite reasonable for this bulk sample with small inhomogeneity. Recently, Langbein et al., observed that the $X X_{A A}^{*}$ state moves from the $2 X_{A}$-continuum edge to higher energetic region, increasing inhomogeneity due to the well-width fluctuation in GaAs quantum wells. ${ }^{23}$ As compared with Fig. 1(b), the contribution in the vicinity of $X_{A}-X X_{A A}^{*}$ and $X_{B}-X X_{B B}^{*}$ changes significantly, whereas the central energy of the $X_{A}-X X_{A A}$ spectrum does not change when the delay time $\tau_{12}$ varies from negative to positive values. The effect can be explained by $X X_{A B}$ that will be discussed later.

For colinear polarization $(\uparrow, \uparrow)$, the $X_{A}-X X_{A A}$ component can appear also in accordance with the polarization selection rules, and in fact, the small peak was observed in the spectra though the component is much smaller compared with the signal at $G-X_{A}$ transition. Same as the case for $(\uparrow, \longrightarrow)$, $X_{A}-X X_{A A}^{*}$ may contribute to similar extent at the energy of $X_{A}$. In addition, two main peaks at $X_{A}$ and $X_{B}$ are found to be blue shifted slightly from the $\left(\sigma_{+}, \sigma_{+}\right)$spectra and is not shifted from the $(\uparrow, \longrightarrow)$ spectra. The blue shift can be explained by the spin-dependent $X_{A}-X_{B}$ interaction (i.e., $X X_{A B}^{*}$ ) and therefore is seen also in $(\uparrow, \longrightarrow)$ spectra. Here, the $X_{A}-X_{B}$ interaction means the correlation via PSF in electronic spin states of $X_{A}$ and $X_{B}$. For example, as shown in Fig. 2, the states $X_{A}$ and $X_{B}$ with $J_{z}=1$ can be represented as $\mid 3 / 2$, $-3 / 2,1 / 2,-1 / 2\rangle$ and $|1 / 2,-1 / 2,1 / 2,1 / 2\rangle$ in $\left|J^{v}, m_{J}^{v}, J^{c}, m_{J}^{c}\right\rangle$.

While these $X_{A}$ and $X_{B}$ with the same exciton spins that are created in a broadband $\left(\sigma_{+}, \sigma_{+}\right)$excitation, have different electronic spin states and no PSF occurs in any state, PSF occurs in the electron spins in $(\uparrow, \uparrow)$ and $(\uparrow, \rightarrow)$ excitations because $X_{A}$ and $X_{B}$ with different exciton spins are created simultaneously. Thus, with opposite exciton spins, the attractive force works between the same species $\left(X_{A}-X_{A}\right.$ and $X_{B}-X_{B}$ ) and makes the bound biexciton $X_{A A}$ and $X_{B B}$, and the repulsive force works between the different species $\left(X_{A}-X_{B}\right)$. Conversely, with the same exciton spins, the attractive force works between $X_{A}$ and $X_{B}$ and the repulsive force works between the same species. This $X_{A}-X_{B}$ interaction can be considered as the effect of $X X_{A B}^{*}$. This effect gives rise also to the phase shift of $X_{A}-X_{B}$ quantum beats in the temporal evolution as seen in Fig. 3. The influence of $X_{A}-X_{B}$ interaction on the phase shift of $X_{A}-X_{B}$ quantum beats was analyzed by Aoki et al. by using weakly interacting Boson model. ${ }^{11}$ 
Figures 1(c) and 1(d) show the examples of the decomposition of the FWM spectra. The fitting was performed assuming that Gaussian line shape in accordance with the inhomogeneous broadening. As a result, for all polarization configurations, $(\uparrow, \uparrow),(\uparrow, \rightarrow)$, and $\left(\sigma_{+}, \sigma_{+}\right)$, two weak and relatively broad components appear at the energies by $\sim 1.4 \mathrm{meV}$ in the low-energy side of $X_{A}$ and $X_{B}$. The components are indicated by the shade in the figures. Without these components, for example, the trough between $X_{A}$ and $X_{B}$ for $\left(\sigma_{+}, \sigma_{+}\right)$or the small peak at the wing of $X_{B}$ is not possible to be sufficiently reproduced. Those components also appeared in the FWM spectra at negative $\tau_{12}$ and for all polarization configurations.

The fact, those components are observed at the lowerenergy side apart by the same amount from both $X_{A}$ and $X_{B}$ resonances regardless of the polarization configurations and the sign of the delay times, leads to the presence of the heterobiexciton $X X_{A B}$ that consists of $X_{A}$ and $X_{B}$ as a bound biexciton state. The heterobiexciton has been observed also in a $100-\AA$-ZnSe single-quantum well by FWM (Ref. 24 ) and a bulk $\mathrm{ZnO}$ by two-photon reabsorption spectroscopy. ${ }^{25}$ The bound biexcitons that consist of the same species, $X X_{A A}$ and $X X_{B B}$, have paired electron spins as well as hole spins, and $J_{z}=0$. On the other hand, $X X_{A B}$ has different hole spins and opposite electron spins, and therefore their $J_{z}$ are \pm 2 as shown in Fig. 2. Reflecting the low density of $X_{B}$ compared with $X_{A}$, the $X X_{A B}$ component in the lower wing of $X_{B}$ is larger than that in the lower wing of $X_{A}$. From the observed spectra, the binding energy of $X X_{A B}$ is $1.4 \mathrm{meV}$ that corresponds to the period of $\sim 3 \mathrm{ps}$ if quantum beats between $X_{\alpha}-X_{A B}$ and $X_{\alpha}-X_{A B}^{*}(\alpha=A, B)$ occurs. Unfortunately, small population of $X X_{A B}$ as well as the long period compared with the phase relaxation time smear the beating in temporal domain. In general, the biexciton binding energy has the tendency that the binding energy is increasing with decreasing electron-hole mass ratio $m_{e}^{*} / m_{h}^{*}$, and the measured value is on this tendency.

The precise interpretation of XXC requires the formalisms to handle $n$-particle correlations such as dynamics controlled truncation scheme (DCTS), ${ }^{26}$ where the coupled equations of motion of one-pair and two-pair correlations at least have to be computed. Since it is difficult to carry out for our experimental situation as shown in Fig. 2, instead we will survey the temporal behavior qualitatively in the light of the XXC, particularly, the $X_{A}-X_{B}$ interaction which is nothing but $X X_{A B}^{*}$.

Figure 3 shows the spectrally resolved FWM traces as a function of $\tau_{12}$ at the energy of $X_{A}$. For the comparison, the signals for $\left(\sigma_{+}, \sigma_{+}\right)$at $X_{B}$ energy (dashed line) is also depicted. The signal decay for $\tau_{12}>0$ is well fitted with the time constant of 0.66 ps by using a suitable equation for intermediate inhomogeneous broadening and the decay time is nearly the same for all polarization configurations. Fundamentally, the signal for $\tau_{12}>0$ shows the decay of the firstorder polarization created by $\boldsymbol{k}_{1}$ pulse that drives the $G-X_{A}$ transition. The signal intensities in $\left(\sigma_{+}, \sigma_{+}\right)$and $(\uparrow, \uparrow)$ are approximately one order of magnitude stronger than that in $(\uparrow, \rightarrow)$ (not shown here) at $\tau_{12}>0$, which shows that the
EID-induced process is strongly suppressed for $(\uparrow, \rightarrow)$. Thus, $\mathrm{XXC}\left(X X_{A A}^{*}\right.$ and $X X_{A B}^{*}$ in this case) contributes strongly to the FWM signal even at $\tau_{12}>0$. This signal intensity ordering observed as well as in the FWM spectra has been measured generally in GaAs-based materials, which is determined by the magnitudes of $X X_{A A}^{*}, X X_{A B}^{*}$ and the phase between them. All the FWM signals indicate the clear beating character. The beating for $\left(\sigma_{+}, \sigma_{+}\right)$and $(\uparrow, \uparrow)$ signals is well reproduced with the frequency of $1.31 \mathrm{THz}$, corresponding to $5.4 \mathrm{meV}$. The energy coincides well to the energy difference between $X_{A}$ and $X_{B}(5.3 \mathrm{meV})$ from linear spectroscopies. From the beat period and $\pi$-phase-shift character for $(\uparrow, \rightarrow)$ against $\left(\sigma_{+}, \sigma_{+}\right)$and $(\uparrow, \uparrow)$ signals, the beating can be assigned to $X_{A}-X_{B}$ quantum beat. The initial phase of the quantum beat in $\left(\sigma_{+}, \sigma_{+}\right)$is shifted by $0.4 \pi$ compared with the beat in $(\uparrow, \uparrow)$. For $X_{B}$ (not shown in Fig. 3), the phase shift has an opposite sign $(\sim-0.15 \pi)$. This spindependent $X_{A}-X_{B}$ interaction induces the phase shift as well as the aforementioned energy shift in the FWM spectra [Fig. $1(\mathrm{a})]$.

For $\tau_{12}<0$, the signal should be entirely due to the Coulomb-induced nonlinearities. It is expected that the bound biexcitons, such as $X X_{A A}, X X_{B B}$, and $X X_{A B}$, will make a more noticeable contribution in processes where twophoton transitions are active, i.e., for negative delay times, and their continua or their unbound biexcitons play a minor role. But this is not the case. In ideal case, because the pulse with the wave vector $\boldsymbol{k}_{2}$ arrives first at the sample for $\tau_{12}$ $<0$ and the contribution to signal generation process is in second order as shown in the inset of the figure, the signal generation due to PSF is suppressed for all polarizations. Instead, two-photon coherences (TPC's) created ${ }^{2}$ by two $\boldsymbol{k}_{2}$ photons dominate the dynamics of the system. Since the twoexciton state as a source of XXC is created by this twophoton transition, it cannot emit light and builds up until the $\boldsymbol{k}_{1}$ pulse arrives and triggers the FWM emission. The slow rise of the signals that are very similar to the rise time of $X_{A}-X X_{A A}$, therefore, suggests that the signals are related to the TPC's. Especially, for $\left(\sigma_{+}, \sigma_{+}\right)$polarizations where the creation of $X X_{A A}$ is inhibited, the slow rise is unexpected without XXC since the signal should rise with a shorter time constant than half the decay time for $\tau_{12}>0$ at least in the case that any XXC's do not work. $X X_{A A}^{*}$ and $X X_{A B}^{*}$ contribute to the signal and the clear beating indicates the existence of $X X_{A B}^{*}$. For $\left(\sigma_{+}, \sigma_{+}\right)$, the beating period for $\tau_{12}<0$ is almost the same as that for $\tau_{12}>0$ and there is no phase jump around $\tau_{12}=0$. Under no signal generation pathway via PSF, this means that the beating originates from $X X_{A B}^{*}$-TPC-induced interference between the transitions $X_{A}-X X_{A B}^{*}$ and $X_{B}-X X_{A B}^{*}$. A pronounced beating for $(\uparrow, \uparrow)$ has a higher contrast than that for $\tau_{12}>0$ and indicates the significant $X X$-TPC and $X X^{*}$-TPC contributions. The beating originates from the interference between the signals induced by the TPC's and the XXC is attributed mainly to the correlation between $X X_{A A}^{*}$ and $X X_{A B}^{*}$. For the quantitative explanation, the computational challenge for this system are currently proceeded. 


\section{SUMMARY}

In summary, we have investigated the FWM responses of the exciton-biexciton system in a free-standing GaN. The spectrally resolved FWM signals were discussed in terms of biexciton formation and its contribution were explained qualitatively. The formation of not only $A$ biexcitons $X X_{A A}$ $\left(\Delta E_{b}^{A A}=5.3 \mathrm{meV}\right)$ but also a heterobiexciton $X X_{A B}\left(\Delta E_{b}^{A B}\right.$ $=1.4 \mathrm{meV}$ ) has been identified by polarization selection

*Electronic address: adachi-s@eng.hokudai.ac.jp

'CREST, Japan Science and Technology Corporation, Kitaku, Sapporo 060-8628, Japan.

${ }^{*}$ Also at Integrative Bioscience and Biomedical Engineering, Graduate School of Science and Engineering, Waseda University, Shinjuku, Tokyo 169-8555, Japan.

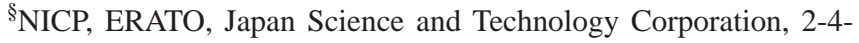
6 Fujimi, Chiyoda-ku, Tokyo 102-0071, Japan and Photodynamics Research Center, RIKEN, Aoba, Sendai, Miyagi 980-0845, Japan.

${ }^{1}$ A.L. Ivanov, H. Wang, J. Shah, T.C. Damen, L.V. Keldysh, H. Haug, and L.N. Pfeiffer, Phys. Rev. B 56, 3941 (1997).

${ }^{2}$ K.B. Ferrio and D.G. Steel, Phys. Rev. B 54, R5231 (1996).

${ }^{3}$ E.J. Mayer, G.O. Smith, V. Heuckeroth, J. Kuhl, K. Bott, A. Schulze, T. Meier, S.W. Koch, P. Thomas, R. Hey, and K. Ploog, Phys. Rev. B 51, 10909 (1995).

${ }^{4}$ K. Bott, O. Heller, D. Bennhardt, S.T. Cundiff, P. Thomas, E.J. Mayer, G.O. Smith, R. Eccleston, J. Kuhl, and K. Ploog, Phys. Rev. B 48, 17418 (1993).

${ }^{5}$ A.E. Paul, J.A. Bolger, A.L. Smirl, and J.G. Pellegrino, J. Opt. Soc. Am. B 13, 1016 (1996).

${ }^{6}$ H.P. Wagner, A. Schätz, W. Langbein, J.M. Hvam, and A.L. Smirl, Phys. Rev. B 60, 4454 (1999).

${ }^{7}$ W. Langbein, T. Meier, S.W. Koch, and J.M. Hvam, J. Opt. Soc. Am. B 18, 1318 (2001).

${ }^{8}$ P. Kner, W. Schäfer, R. Lövenich, and D.S. Chemla, Phys. Rev. Lett. 81, 5386 (1998).

${ }^{9}$ A.J. Fischer, W. Shan, G.H. Park, J.J. Song, D.S. Kim, D.S. Yee, R. Horning, and B. Goldenberg, Phys. Rev. B 56, 1077 (1997).

${ }^{10}$ S. Pau, J. Kuhl, F. Scholz, V. Haerle, M.A. Khan, and C.J. Sun, Phys. Rev. B 56, R12718 (1997).

${ }^{11}$ T. Aoki, G. Mohs, M. Kuwata-Gonokami, and A.A. Yamaguchi, Phys. Rev. Lett. 82, 3108 (1999). rules. The unbound $X X_{A B}^{*}$ is essential for the $X_{A}-X_{B}$ interaction and contributes more significantly to the FWM signal generation rather than the bound $X X_{A B}$ biexcitons, especially, for $\left(\sigma_{+}, \sigma_{+}\right)$and $(\uparrow, \longrightarrow)$ polarizations.

\section{ACKNOWLEDGMENTS}

S.A. acknowledges support by Casio Science Promotion Foundation, TEPCO Research Foundation, NSGF and SCAT.

${ }^{12}$ K. Hazu, A. Shikanai, T. Sota, K. Suzuki, S. Adachi, S.F. Chichibu, and T. Mukai, Phys. Rev. B 65, 195202 (2002).

${ }^{13}$ R. Zimmermann, A. Euteneuer, J. Möbius, D. Weber, M.R. Hofmann, W.W. Rühle, E.O. Göbel, B.K. Meyer, H. Amano, and I. Akasaki, Phys. Rev. B 56, R12722 (1997).

${ }^{14}$ K. Kyhm, R.A. Taylor, J.F. Ryan, T. Aoki, M. Kuwata-Gonokami, B. Beaumont, and P. Gibart, Phys. Status Solidi B 228, 475 (2001).

${ }^{15}$ For a review, S. Nakamura and G. Fasol, The Blue Laser Diode (Springer-Verlag, Heidebelg, 1997).

${ }^{16}$ A. Usui, H. Sunakawa, S. Sakai, and A.A. Yamaguchi, Jpn. J. Appl. Phys. 36, L899 (1997).

${ }^{17}$ S. Nakamura, M. Senoh, S. Nagahama, N. Iwasa, T. Yamada, T. Matsushita, H. Kiyoku, Y. Sugimoto, T. Kozaki, H. Umemoto, M. Sano, and K. Chocho, Jpn. J. Appl. Phys. 37, L309 (1998).

${ }^{18}$ M. Suzuki, T. Uenoyama, and A. Yanase, Phys. Rev. B 52, 8132 (1995).

${ }^{19}$ J. Shah, Ultrafast Spectroscopy of Semiconductors and Semiconductor Nanostructures (Springer-Verlag, New York, 1998).

${ }^{20}$ K. Torii, T. Deguchi, T. Sota, K. Suzuki, S. Chichibu, and S. Nakamura, Phys. Rev. B 60, 4723 (1999).

${ }^{21}$ H. Wang, K. Ferio, D.G. Steel, Y.Z. Hu, R. Binder, and S.W. Koch, Phys. Rev. Lett. 71, 1261 (1993).

${ }^{22}$ Y.Z. Hu, R. Binder, S.W. Koch, S.T. Cundiff, H. Wang, and D.G. Steel, Phys. Rev. B 49, 14382 (1994).

${ }^{23}$ W. Langbein and J.M. Hvam, Phys. Status Solidi A 190, 167 (2002).

${ }^{24}$ H.P. Wagner, W. Langbein, and J.M. Hvam, Phys. Rev. B 59, 4584 (1999).

${ }^{25}$ J.M. Hvam, G. Blattner, M. Reuscher, and C. Klingshirn, Phys. Status Solidi B 118, 179 (1983).

${ }^{26}$ V.M. Axt and A. Stahl, Z. Phys. B: Condens. Matter 93, 195 (1994). 